\title{
Edge phase transitions of the tricritical Potts model in two dimensions
}

\author{
Youjin Deng ${ }^{1, *}$ and Henk W. J. Blöte ${ }^{1,2}$ \\ ${ }^{1}$ Faculty of Applied Sciences, Delft University of Technology, P. O. Box 5046, 2600 GA Delft, The Netherlands \\ ${ }^{2}$ Lorentz Institute, Leiden University, P. O. Box 9506, 2300 RA Leiden, The Netherlands
}

(Received 2 October 2004; published 10 February 2005)

\begin{abstract}
Using Monte Carlo techniques and finite-size analysis, we investigate several two-dimensional lattice models with open edges, including the Blume-Capel model and the $q=1$ and 3 Potts models with vacancies. At bulk tricriticality, we find that the open edges are dominated by the vacancies when the surface coupling $K_{s}$ and the chemical potential $D_{s}$ of the vacancies assume the bulk values. When $K_{s}$ and/or $D_{s}$ is sufficiently enhanced, an edge phase transition takes place, beyond which spontaneous one-dimensional order occurs on the edges. Edge phase transitions can also be induced by a surface magnetic field $H_{s}$. We numerically determine a number of edge critical exponents and derive phase diagrams in terms of $K_{s}, D_{s}$, and $H_{s}$. In the low-temperature region, we observe first-order transitions when $K_{s}$ and $D_{s}$ are varied; the associated hysteresis loops of surface quantities are remarkably asymmetric. Some further insight into these edge transitions is provided by the exact equivalence of the tricritical $q=1$ Potts model and the Ising model.
\end{abstract}

DOI: 10.1103/PhysRevE.71.026109

PACS number(s): 05.50.+q, 64.60.Cn, 64.60.Fr, 75.10.Hk

\section{INTRODUCTION}

While theoretical physicists frequently study phase transitions in systems with periodic boundary conditions, in reality systems generally have surfaces. Thus, there may be a need to consider the effects due to the presence of surfaces. For a $d$-dimensional system containing $L^{d}$ atoms, the relative fraction of atoms at or near a surface is of order $1 / L$, and hence for large $L$ it is usually justified to neglect the surface effects on bulk properties of the material. However, near a phase transition, correlations become long ranged, so that relatively small perturbations can produce large responses. Therefore, surface effects can become significant, and in many cases they cannot be ignored. Indeed surface phase transitions have been the subject of considerable research interest in the past decades [1-11]. Many theoretical and numerical methods have been developed, including mean-field approximations, high- and low-temperature expansions, renormalization group techniques, conformal field theory, and Monte Carlo simulations, etc.

Most of these results apply to three-dimensional systems, and in this context, we briefly review surface critical phenomena of the Ising model on a simple-cubic lattice with two open surfaces in the $z$ direction and periodic boundary conditions in the $x y$ plane $[1,4,8,10]$. The Hamiltonian of this system can be divided into two parts: bulk terms and surface terms, i.e.,

$$
\mathcal{H} / k_{\mathrm{B}} T=-K \sum_{i j}^{\mathrm{b}} s_{i} s_{j}-H \sum_{k}^{\mathrm{b}} s_{k}-K_{s} \sum_{l m}^{\mathrm{s}} s_{l} s_{m}-H_{s} \sum_{n}^{\mathrm{s}} s_{n} .
$$

The spins assume values \pm 1 , and interactions occur between nearest-neighbor spins. The first two sums account for the

\footnotetext{
*Present address: Laboratory for Material Science, Delft University of Technology, Rotterdamseweg 137, 2628 AL Delft, The Netherlands.
}

bulk, and the last two sums involve spins on the open surfaces. For a finite cube with linear size $L$, the surface terms concern an area $2 L^{2}$, because there are surfaces both at $z$ $=0$ and at $z=L$.

In three dimensions, exact information is scarce about the bulk critical behavior of the Ising model described by Eq. (1), so that investigations have to depend on approximations. Nevertheless, accurate information has been obtained. For instance, it has been determined [12] that the bulk critical point is $K=K_{c}=0.22165455(3)$ and $H=H_{c}=0$, and the thermal and magnetic renormalization exponents are $y_{t}$ $=1.5868(3)$ and $y_{h}=2.4816(1)$, respectively. Surface critical phenomena in this magnetic system (1) are now also well analyzed $[1,4,8,10]$. In the absence of magnetic fields $H$ $=H_{s}=0$ and for ferromagnetic couplings $K \geqslant 0$ and $K_{s} \geqslant 0$, the phase diagram is sketched in Fig. 1. In the hightemperature region, i.e., the bulk couplings $K<K_{c}$, the bulk

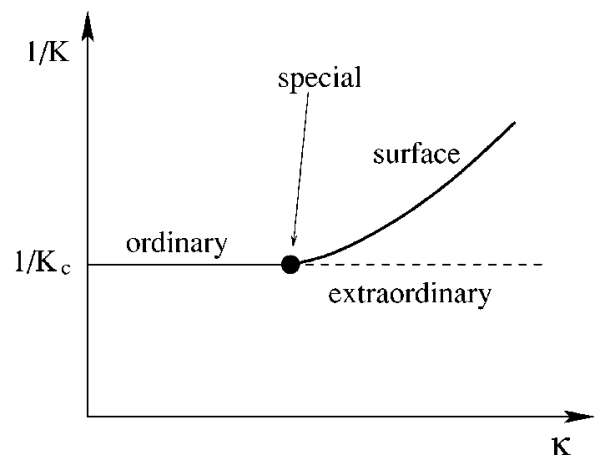

FIG. 1. Sketch of the surface phase transitions of the Ising model in three dimensions. The vertical axis is the bulk temperature $1 / K$, and the parameter $\kappa=\left(K_{S}-K\right) / K$ in the horizontal axis represents the enhancement of the surface couplings. The "surface," the "ordinary," and the "extraordinary" phase transitions are represented by the thick solid, the thin solid, and the dashed lines, respectively. The lines meet in a point, shown as the black circle, which is referred to as the "special" phase transition. 
is in the paramagnetic state ("disordered"), so that the bulk correlations remain finite. However, phase transitions can still occur on the open surfaces when the surface couplings $K_{s}$ are varied. These transitions, referred to as the "surface transitions", are shown as the curved solid line in Fig. 1. Apparently, they belong to the same universality class as Onsager's Ising model [13] in two dimensions, so that the thermal and magnetic exponents are [13] $y_{t s}=1$ and $y_{h s}$ $=15 / 8$, respectively. At the bulk critical point $K=K_{c}$, the line of surface transitions terminates at a point $\left(K_{c}, K_{s c}\right)$, which acts as a multicritical point. For relatively small surface couplings $K_{s}<K_{s c}$, both the bulk and the surfaces undergo a second-order phase transition at $K=K_{c}$ when $K$ is varied. However, for larger surface couplings $K_{s}>K_{s c}$, the surfaces become ferromagnetic at a smaller bulk coupling $K<K_{c}$, so that the bulk transition $K=K_{c}$ occurs in the presence of spontaneous surface order. Along the bulk critical line $K=K_{c}$, the phase transitions for $K_{s}<K_{s c}, K_{s}=K_{s c}$, and $K_{s}>K_{s c}$ are referred to as the "ordinary," the "special," and the "extraordinary" transitions, respectively. In order to describe the scaling aspects of these surface transitions, besides the bulk exponents $y_{t}$ and $y_{h}$, additional surface critical exponents are also needed. The ordinary and the extraordinary transitions have one additional relevant surface magnetic scaling field; both the surface thermal and magnetic scaling fields are relevant at the special transition. We denote the corresponding exponents as $y_{h s}^{(\mathrm{o})}, y_{h s}^{(\mathrm{e})}, y_{t s}^{(\mathrm{s})}$, and $y_{h s}^{(\mathrm{s})}$ respectively, where the superscripts (o), (e), and (s) are for the ordinary, the extraordinary, and the special transitions, respectively. In an analogy with the bulk ones $y_{t}$ and $y_{h}$, exact values of these surface exponents are unavailable. It has been numerically determined $[1,4,8,9,14]$ that $y_{h s}^{(\mathrm{o})}=0.737(5), y_{t s}^{(\mathrm{s})}=0.94(6)$, and $y_{h s}^{(\mathrm{s})}$ $=1.62(2)$.

The present paper investigates the surface effects on a number of two-dimensional systems. However, in this case, the "surfaces" are just one-dimensional edges. Since onedimensional systems with short-range interactions are known not to order for any nonzero temperature, the "surface transitions" occurring at $K<K_{c}$ simply cannot exist on open edges of two-dimensional systems. It may then seem natural that no spontaneous edge order can occur without a longranged ordered bulk. In other words, in two dimensions, it may be expected that only the ordinary transition exists on the one-dimensional surfaces. It has further been argued [1] that the surface dimensionality $d=2$ is the lower critical dimensionality for the special, the surface, and the extraordinary transitions. This is consistent with exact results for the Ising model in two dimensions. Exact calculations of surface effects in this model $[15,16]$ were not restricted to the critical region but covered in the entire temperature range. At the bulk criticality, it was shown that, for any finite surface coupling $K_{s}$, the transition on the open edges is just the ordinary transition. The corresponding surface magnetic exponent is $y_{h s}^{(\mathrm{o})}=1 / 2[15,16]$, different from the bulk exponent $y_{h}$ $=15 / 8$ [13]. The surface magnetization density $m_{s}$ and the surface susceptibility $\chi_{s}$ at the ordinary transition appear to be of logarithmic nature $[15,16]$, i.e.,

$$
m_{s}\left(H_{s}\right) \propto H_{s}\left|\ln H_{s}\right| \quad\left(K=K_{c},\left|H_{s}\right| \ll 1\right)
$$

and

$$
\chi_{s}(t) \propto|\ln | t|| \quad\left(H_{s}=0,|t|=\left|K-K_{c}\right| \ll 1\right),
$$

where $\chi_{s}$ is defined as $\partial m_{s} / \partial H_{s}$.

The statement that only ordinary transitions occur on the edges can be generalized to the critical branch of the $q$-state Potts model in two dimensions. For a review of the Potts model, see Ref. [17]. For this model, the nature of the bulk critical singularities is now well established. This is mostly due to exact calculations $[19,18]$, Coulomb gas theory [20], and conformal field theory [3]. In the context of the Coulomb gas theory [20], a sequence of universal exponents can be exactly expressed in terms of a single parameter $g$, i.e., the coupling strength of the Coulomb gas. The parameter $g$ satisfies $q=2+2 \cos (g \pi / 2)$, with $2 \leqslant g \leqslant 4$ and $4 \leqslant g \leqslant 6$ for the critical and the tricritical branch of the Potts model, respectively. The leading thermal and magnetic exponents of the Potts model are $[20,21] y_{t}=3-6 / g$ and $y_{h}=(g+2)(g+6) / 8 g$, respectively. For the ordinary surface transition of the critical Potts model, Cardy [5-7] employed boundary conformal field theory, and expressed the surface magnetic exponent $y_{h s}^{(\mathrm{o})}$ in terms of the bulk thermal exponent $y_{t}$ as

$$
y_{h s}^{(\mathrm{o})}=2-3 /\left(3-y_{t}\right)=2-g / 2 \quad(2 \leqslant g \leqslant 4) .
$$

A remarkable feature of Eq. (4) is that $y_{h s}^{(\mathrm{o})}$ is a decreasing function of the Coulomb gas coupling $g$. In particular, for the $q=4$ Potts model $(g=4)$, Eq. (4) yields $y_{h s}^{(\mathrm{o})}=0$, so that the surface magnetic scaling field is marginal. It seems natural that Eq. (4) can also be applied to $g>4$, just as the above expressions for the bulk exponents $y_{t}$ and $y_{h}[20,21]$. This application then yields that the surface magnetic scaling field is irrelevant for the tricritical Potts model. On the other hand, it is known that, near a second-order transition, the strength of critical fluctuations and the sensitivity to perturbations are reflected by the magnitudes of the critical exponents $y_{t}$ and $y_{h}$. For the Potts model, $y_{t}=3-6 / g$ is an increasing function of $g$, and, for $0<q<4, y_{h}$ is larger on the tricritical branch than on the critical one. Thus, one might naively expect that the surface effects, including that of the surface magnetic field $H_{s}$, become stronger as $g$ increases. Further exploration of this paradox seems justified.

Recently, boundary conformal field theory has received considerable research interest [22-27]. In the context of statistical physics, this has been applied to the tricritical Ising model in two dimensions. This model is considered to correspond with an integral scattering theory of massive kinks [22], and it preserves superconformal symmetry. By means of factorizable $S$ matrix, fusion rules, and symmetry arguments, various boundary operators were conjectured [23] and the corresponding renormalization flows were constructed. A physical interpretation of these boundary phenomena was then provided by Affleck [24], indicating the possible emergence of spontaneous edge order if the bulk is in the tricritical state. Moreover, this scenario has been numerically confirmed in Ref. [28].

The present paper extends the work in Ref. [28]. First, as a direct illustration of the existence of the edge transitions in tricritical Potts models in two dimensions, we make use of the exact equivalence of the dilute $q=1$ Potts model with the Ising model in a magnetic field [29]. Thus, the exact infor- 
mation about the edge critical phenomena in the latter model can be reformulated in the language of the former model. Then, using suitable Monte Carlo methods, we simulate the Blume-Capel (BC) model [30,31] and the $q=3$ Potts model with vacancies. From the finite-size analysis of the numerical data, we derive a number of edge phase diagrams in terms of surface parameters, and determine several surface critical exponents.

The outline of this paper is as follows. Section II describes the surface phenomena of the dilute $q=1$ Potts model, as derived from the known properties of the Ising model in a magnetic field. In Secs. III and IV, Monte Carlo results are presented for the surface transitions of the BlumeCapel model and the tricritical $q=3$ Potts model, respectively. A brief discussion is given in Sec. V.

\section{DILUTE ONE-STATE POTTS MODEL}

The dilute Potts model is obtained by including vacancies in the corresponding "pure" Potts model. On the $L \times L$ square lattice with periodic boundary conditions, to which we shall refer as the torus geometry, the Hamiltonian of the dilute $q$-state model reads

$$
\begin{aligned}
\mathcal{H}_{\mathrm{P}} / k_{\mathrm{B}} T= & -K \sum_{x, y=1}^{L}\left(1-\delta_{\sigma_{x, y}, 0}\right)\left(\delta_{\sigma_{x, y}, \sigma_{x+1, y}}+\delta_{\sigma_{x, y}, \sigma_{x, y+1}}\right) \\
& -D \sum_{x, y=1}^{L} \delta_{\sigma_{x, y}, 0}
\end{aligned}
$$

where the lattice site is occupied by a vacancy $\sigma=0$ or a Potts variable with $\sigma=1,2, \ldots, q$. Nonzero couplings $K$ occur only between Potts variables, and the chemical potential $D$ controls the concentration of the vacancies. In Eq. (5), we have introduced the subscript $\mathrm{P}$ to represent periodic boundary conditions. For the special case $q=1$ Eq. (5) reduces, apart from a constant, to

$$
\mathcal{H}_{\mathrm{P}} / k_{\mathrm{B}} T=-K \sum_{x, y=1}^{L} \sigma_{x, y}\left(\sigma_{x+1, y}+\sigma_{x, y+1}\right)+D \sum_{x, y=1}^{L} \sigma_{x, y}(\sigma=0,1) .
$$

For $D \rightarrow-\infty$, the vacancies are excluded, and the first sum of Eq. (6) is just a constant. Nevertheless, the random-cluster representation of Hamiltonian (6) corresponds with the bondpercolation model with bond-occupation probability $p=1$ $-\exp (-K)$, so that Eq. (6) still describes percolation phenomena. In the presence of vacancies, Eq. (6) describes a correlated dilute bond-percolation model, which can be transformed into the Ising model in a magnetic field. This follows from substitution of $s=2 \sigma-1$ in Eq. (6), which yields

$$
\begin{aligned}
\mathcal{H}_{\mathrm{P}}^{(\mathrm{i})} / k_{\mathrm{B}} T= & -K^{(\mathrm{i})} \sum_{x, y=1}^{L} s_{x, y}\left(s_{x+1, y}+s_{x, y+1}\right) \\
& -H^{(\mathrm{i})} \sum_{x, y=1}^{L} s_{x, y}(s= \pm 1),
\end{aligned}
$$

with the relations

$$
K^{(\mathrm{i})}=K / 4 \quad \text { and } \quad H^{(\mathrm{i})}=-D / 2+K,
$$

where the superscript (i) refers to the Ising model. In the absence of a magnetic field $H^{(\mathrm{i})}$, the Ising model (7) has a critical point at $K^{(\mathrm{i})}=K_{c}^{(\mathrm{i})}=\ln (1+\sqrt{2}) / 2$ [13]. This point is not percolationlike; it serves as the tricritical point of the $q=1$ Potts system (6). Equation (8) yields the tricritical point as $K=K_{t}=2 \ln (1+\sqrt{2})$ and $D=D_{t}=4 \ln (1+\sqrt{2})$; the up-down symmetry of Ising spins implies that the tricritical vacancy density is $\rho=1 / 2$. Further, it follows from Eq. (8) that the leading and the subleading thermal exponents of the tricritical Potts model are equal to the magnetic and the thermal exponents of the Ising model, respectively, so that $y_{t 1}$ $=15 / 8$ and $y_{t 2}=1$. The leading magnetic exponent is known as $y_{h 1}=187 / 96$ [20]. In the low-temperature region $K^{(\mathrm{i})}$ $>K_{c}^{(i)}$, the Ising model undergoes a first-order phase transition when the magnetic field $H^{(\mathrm{i})}$ changes sign. In other words, the dilute $q=1$ Potts model (6) has a line of first-order phase transitions at $D=2 K$ for $K>K_{t}$.

Because of the attraction between the vacancies, the dilute $q=1$ model (6) is different from the conventional site-bondpercolation problem [32]. In the latter system, the vacancies are randomly distributed over the lattice sites, and then bonds are placed with probability $0 \leqslant p \leqslant 1$ between all nearest-neighboring occupied sites. Apart from that, sites and bonds are uncorrelated. A limiting case is the "pure" sitepercolation model, in which the bond-occupation probability is 1 . This model is still in the percolation universality, so that no tricritical point exists for the conventional site-bondpercolation problem. In contrast, for a correlated dilute $q$-state Potts model described by Eq. (5), it has been found $[29,33]$ that the tricritical point occurs for any value in the continuous range $0 \leqslant q \leqslant 4$.

In order to investigate the surface effects, we define the correlated percolation model (6) on an open cylinder, i.e., the $L \times L$ square lattice with periodic and free boundary conditions in the $x$ and the $y$ directions, respectively. As for the three-dimensional case, the surface couplings $K_{s}$ and the chemical potential $D_{s}$ can assume different values from those in the bulk. The Hamiltonian $\mathcal{H}_{\mathrm{O}}$ on the open cylinder can be written as the sum of $\mathcal{H}_{\mathrm{P}}$ in Eq. (6) and their difference, which reads

$$
\begin{aligned}
\mathcal{H}_{\mathrm{O}} / k_{\mathrm{B}} T-\mathcal{H}_{\mathrm{P}} / k_{\mathrm{B}} T= & K \sum_{x=1}^{L} \sigma_{x, 1} \sigma_{x, L} \\
& -K \kappa_{k} \sum_{x=1}^{L}\left(\sigma_{x, 1} \sigma_{x+1,1}+\sigma_{x, L} \sigma_{x+1, L}\right) \\
& -D \kappa_{d} \sum_{x=1}^{L}\left(\sigma_{x, 1}+\sigma_{x, L}\right)
\end{aligned}
$$

where $\kappa_{k}=K_{s} / K-1$ and $\kappa_{d}=D_{s} / D-1$ represent the enhancements of the surface coupling and the chemical potential, respectively. The subscript $\mathrm{O}$ is for the open cylinder. The sums in the right-hand side of Eq. (9) are only over spins sitting on the edges $y=1$ and $y=L$. Thus, the surface effects can be regarded as containing two parts: the first term in Eq. (9) accounts for the geometric effect due to "missing" neigh- 
bors for spins at the surface layers, and the last two sums describe the enhancements of the surface parameters $K_{s}$ and $D_{s}$. Effectively, the first term serves as a perturbation which decreases the correlations along the lines $y=1$ and $y=L$. After substituting $\sigma=(s+1) / 2$ in Eq. (9), one obtains the Ising model on a cylinder with open ends

$$
\begin{aligned}
\mathcal{H}_{\mathrm{O}}^{(\mathrm{i})} / k_{\mathrm{B}} T-\mathcal{H}_{\mathrm{P}}^{(\mathrm{i})} / k_{\mathrm{B}} T \\
=\frac{K}{4} \sum_{x=1}^{L} s_{x, 1} s_{x, L}-\frac{K}{4} \kappa_{k} \sum_{x=1}^{L}\left(s_{x, 1} s_{x+1,1}+s_{x, L} s_{x+1, L}\right) \\
\quad-H_{s}^{(\mathrm{i})} \sum_{x=1}^{L}\left(s_{x, 1}+s_{x, L}\right),
\end{aligned}
$$

with a surface magnetic field $H_{s}^{(\mathrm{i})}$

$$
H_{s}^{(\mathrm{i})}=\left[K\left(2 \kappa_{k}-1\right)-2 D \kappa_{d}\right] / 4 .
$$

Due to the equivalences of Eqs. (6) and (7), and of Eqs. (9) and (10), the edge transitions of the Ising model can now be reformulated in the language of the dilute $q=1$ Potts model. In the high-temperature region $K>K_{t}$, no transition occurs on the one-dimensional edge. At bulk criticality, the Ising model exhibits an ordinary edge transition at $H_{s}^{(\mathrm{i})}=0$, and the surface magnetic exponent is $y_{h s}^{(\mathrm{o})}=1 / 2[15,16]$. In the context of the tricritical $q=1$ Potts model (9), this means that, as indicated by the relation (11), an edge transition can be induced by varying the surface couplings $K_{s}$ and the chemical potential $D_{s}$. For instance, for the case $\kappa_{d}=0$, the edges of the Potts model are dominated by vacancies $(\sigma$ $=0)$ or by Potts variables $(\sigma=1)$ for $\kappa_{k}<1 / 2$ or $\kappa_{k}>1 / 2$, respectively. Since $K_{s}$ and $D_{s}$ are temperaturelike parameters, we refer to such an edge transition as the "special transition." The surface thermal exponent is simply obtained as $y_{t s}^{(\mathrm{s})}(q$ $=1)=1 / 2$. In the low-temperature region $K>K_{t}$, the bulk of the Potts model (9) is in a two-phase equilibrium along the line $D / K=2$, as discussed above. Therefore, a small perturbation due to an enhancement $\kappa_{k}$ or $\kappa_{d}$ induces a first-order transition, which involves the bulk as well as the edges. Figure 2 sketches the phase diagram of the dilute $q=1$ Potts model (9) for the case $\kappa_{d}=0$.

For the $q=1$ Potts model at bulk tricriticality, Eq. (11) yields a line of "special" critical points in the $\left(\kappa_{k}, \kappa_{d}\right)$ plane, as shown in Fig. 3.

\section{BLUME-CAPEL MODEL}

The previous section indicates that, also in twodimensional systems, special phase transitions can occur. However, the dilute $q=1$ Potts model described by Eq. (9) is only a special case. For instance, the coupling constants and the chemical potential in this Potts model are just the magnetic field in the Ising model. In the following two sections, we shall investigate the Blume-Capel model and the dilute $q=3$ Potts model.

The BC model, also referred to as the spin-1 Ising model, was independently introduced by Blume [30] and Capel [31]. This model can be obtained by including vacancies in the

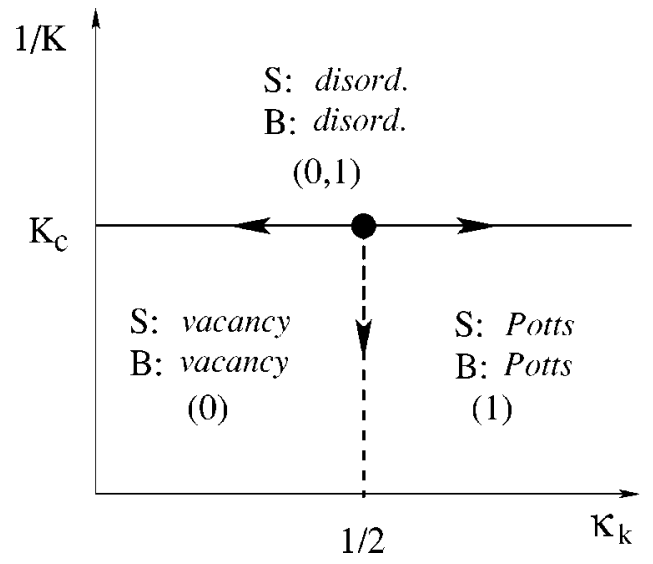

FIG. 2. Edge phase transitions of the dilute $q=1$ Potts model in two dimensions, with $K / D=1 / 2$ and $\kappa_{d}=\left(D_{s}-D\right) / D=0$. For $K$ $<K_{c}$, both the bulk and the surface are in the "disordered" state, and no edge transition occurs. At bulk tricriticality $K=K_{c}$, the variation of $\kappa_{k}=\left(K_{s}-K\right) / K$ yields a "special" edge critical point at $\kappa_{k}$ $=1 / 2$. For $K>K_{c}$, a line of first-order phase transitions, $\kappa_{k}=1 / 2$, separates the phases dominated by the vacancies and by the Potts variables, respectively. Arrows describe the direction of the renormalization flow.

Ising model, and it played an important role in the development of the theory of phase transitions and critical phenomena. In the torus geometry, the Hamiltonian reads

$$
\mathcal{H}_{\mathrm{P}} / k_{\mathrm{B}} T=-K \sum_{\langle i j\rangle} s_{i} s_{j}+D \sum_{k} s_{k}^{2} \quad(s=0, \pm 1) .
$$

When the chemical potential $D$ goes to $-\infty$, the vacancies $s$ $=0$ are excluded, and this model reduces to the spin $-1 / 2$ Ising model. The critical coupling constant $K_{c}(D)$ is an increasing function of $D$, and the critical line $K_{c}(D)$ terminates at the tricritical point $\left(K_{t}, D_{t}\right)$. For $K>K_{t}$, this line continues as a line of first-order phase transitions. The phase diagram of the bulk transitions is sketched in Fig. 4. At the tricritical point, there are four relevant scaling fields; two of them are

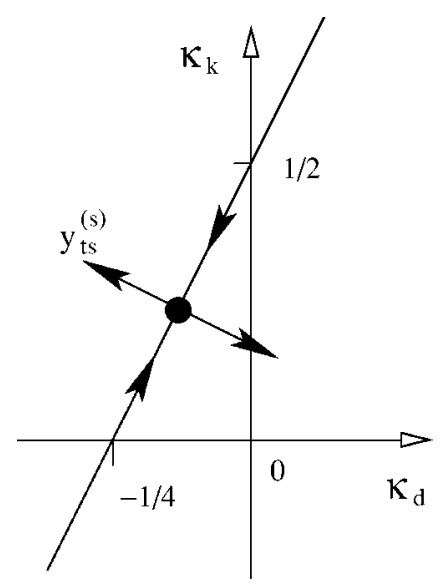

FIG. 3. Line of "special" transitions in the dilute $q=1$ Potts model at bulk tricriticality. The fixed point is shown as a black circle, and the arrows represent the direction of the renormalization flow. 


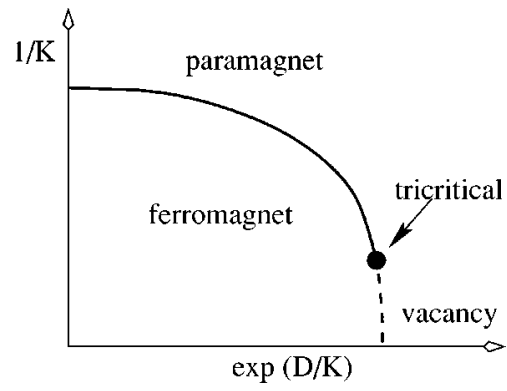

FIG. 4. Sketch of the bulk phase diagram of the BC model. The tricritical point is denoted as the black circle, the second- and the first-order transition lines are represented by the solid and the dashed lines, respectively.

thermal ones and the other two are magnetic ones. In two dimensions, the renormalization exponents are known as $y_{t 1}=9 / 5$ and $y_{t 2}=4 / 5$, and $y_{h 1}=77 / 40$ and $y_{h 2}=9 / 8[20,21]$, respectively. By means of a sparse transfer-matrix technique and finite-size scaling, we located the square-lattice tricritical point [33] as $K_{t}=1.6431759(1)$ and $D_{t}=3.2301797(2)$; the tricritical vacancy density is $\rho_{t}=0.4549506(2)$. This result is obtained from the requirement that both the bulk leading magnetic and energy-energy correlation lengths simultaneously reach their theoretical values. The precision is considered to be sufficient for the present investigation.

On the $L \times L$ open cylinder, as for the dilute $q=1$ Potts model (9), the Hamiltonian of the BC model can be expressed as the sum of the Hamiltonian in the torus geometry and their difference, which reads

$$
\begin{aligned}
\mathcal{H}_{\mathrm{O}} / k_{\mathrm{B}} T-\mathcal{H}_{\mathrm{P}} / k_{\mathrm{B}} T & \\
= & \sum_{x=1}^{L} s_{x, 1} s_{x, L}-K \kappa_{k} \sum_{x=1}^{L}\left(s_{x, 1} s_{x+1,1}+s_{x, L} s_{x+1, L}\right) \\
& +D_{\kappa_{d}} \sum_{x=1}^{L}\left(s_{x, 1}^{2}+s_{x, L}^{2}\right)-H_{s} \sum_{k}\left(s_{x, 1}+s_{x, L}\right),
\end{aligned}
$$

where $H_{s}$ is the surface magnetic field. In the right-hand side of Eq. (13), the first term corresponds with the geometric effect, and the remaining three terms describe the effects of the surface parameters $K_{s}, D_{s}$, and $H_{s}$.

\section{A. Bulk criticality: $K<K_{t}$}

For bulk couplings $K<K_{t}$, the phase transition along the critical line $K_{c}(D)$ is just Ising-like. Thus, in the absence of surface magnetic fields $H_{s}$, only the ordinary transitions occur on the open edges, and the surface magnetic exponent is $y_{h s}^{(\text {o) }}=1 / 2[15,16]$.

\section{B. Bulk tricriticality: $K=K_{t}, D=D_{t}$}

When the bulk is at the tricritical point, the prediction from conformal field theory described by Eq. (4) and the discussions in Sec. II indicate that intriguing phase transitions can occur on the open edges of the BC model.

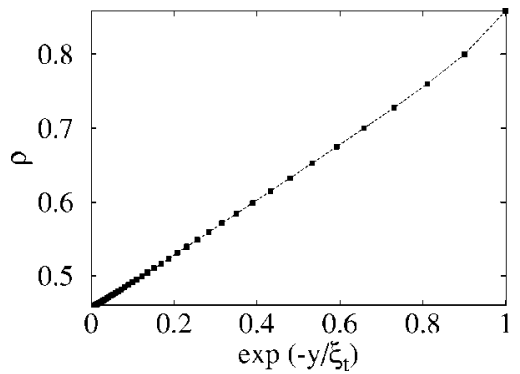

FIG. 5. Exponential decay of the vacancy density $\rho$ along an $L \times 10 L$ cylinder for the tricritical BC model. The system size is chosen as $L=12$, and $r$ represents the distance to one open end. The correlation length used for the horizontal scale is calculated as $\xi_{t}$ $=L /\left(2 \pi X_{t 1}\right)$, where $X_{t 1}=1 / 5$ is the bulk thermal scaling dimension. In the middle of the cylinder, $\rho$ is close to the tricritical value $\rho_{t}$ $=0.4549506$ (2). Deviations from the exponential behavior occur near the edges (right-hand side) and near the middle (not visible on this scale).

\section{Ordinary edge transitions}

As discussed above, the surface effects can be divided into two parts: the geometric effect and the surface enhancements. To study the former effect only, we investigated the tricritical BC model on an open cylinder with circumference $L$ and length $n L$ for $n=10$. We took the surface parameters in Eq. (13) as $\kappa_{k}=\kappa_{d}=0$ and $H_{s}=0$. The system sizes assumed even numbers in the range $8 \leqslant L \leqslant 24$. Simulations used a combination of Wolff and Metropolis steps. The former step flips Ising spins, while the latter step also allows fluctuations of the vacancy density. The vacancy density $\rho$ was sampled along the cylinder. The $\rho$ data for $L=12$ are shown in Fig. 5 . One observes that, without sufficient enhancements of $K_{s}$ and $D_{s}$, the open edges of the tricritical BC model are mainly occupied by the vacancies. This is analogous to the case of the tricritical $q=1$ Potts model.

An explanation of the paradox mentioned after Eq. (4) can be given as follows. As mentioned in Sec. I, the effect of a temperaturelike perturbation is reflected by the bulk thermal exponent $y_{t}$, and thus the geometric effect described by the first term in Eq. (13) also increases as a function of $y_{t}$. For the critical Potts model $(g<4), y_{t}$ is relatively small, so that the edges maintain strong critical correlations. As $g$ increases, however, the density of the vacancies increases and the edge critical correlations become less strong. As a consequence, the surface magnetic field becomes less "effective." On the tricritical branch $(g>4)$, the geometric effect is so large that the edges are dominated by vacancies, and the surface magnetic field becomes irrelevant. We mention that, although the edges have a considerable degree of disorder, and the decay of this disorder into the bulk can be long ranged, the bulk tricritical correlation lengths remain divergent. This is reflected by the asymptotically exponential decay of the vacancy density $\rho$ in Fig. 5, which takes place with the predicted length scale $\xi$. Thus, the bulk transition at $K=K_{t}$ and $D=D_{t}$ occurs in the presence of "disordered" edges. In analogy with the three-dimensional Ising model, we refer to this phase transition as the "ordinary transition."

Under Cardy's well-known conformal mapping [3], the semi-infinite cylinder is be transformed into a semi-infinite 


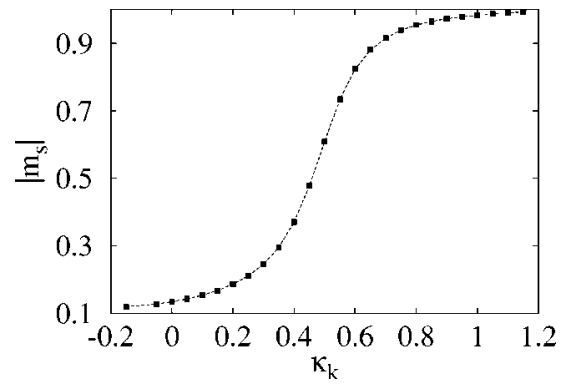

FIG. 6. Absolute value of the edge magnetization $\left|m_{s}\right|$ of the tricritical BC model vs coupling enhancement $\kappa_{k}$. The other surface parameters are $\kappa_{d}=0$ and $H_{s}=0$, and the system size is $L=15$.

plane. The exponential decay of correlations along the cylinder is covariantly transformed into algebraic decay into the bulk of the semi-infinite plane. Thus, the thermal correlation length along the cylinder reads $\xi_{t}=L /\left(2 \pi X_{t 1}\right)$, with the leading thermal scaling dimension $X_{t 1}=2-y_{t 1}=1 / 5[20,21]$. According to the least-squares criterion, we fitted the $\rho$ data by the formula

$$
\begin{aligned}
& \rho(r, L)=\rho_{t}+L^{-2 X_{t 1}}[\Psi(r)+\Psi(n L-r)] \\
& \times\left(a_{0}+a_{1} L^{y_{i}}+a_{2} L^{-2}+\cdots\right),
\end{aligned}
$$

with the function

$$
\Psi(r)=\left(e^{r \pi / L}-e^{-r \pi / L}\right)^{-2 X_{t 1}},
$$

where $r$ is the distance to one of the open ends. A justification of Eqs. (14) and (15) can be found in Refs. [14,34]. The term $\Psi(n L-r)$ in Eq. (14) is due to the symmetry between the positions $r$ and $n L-r$. The parameters $a_{0}, a_{1}$, and $a_{2}$ are unknown constants, and $y_{i}=-1$ is the leading irrelevant thermal exponent of the tricritical $q=2$ universality class [20,21]. For $L \rightarrow \infty$ and $r=L / 2$, the bulk vacancy density $\rho(r, L)$ approaches the tricritical value $\rho_{t}=0.4549506(2)$ [33]. We fixed the values of $y_{i}$ and $\rho_{t}$, and discarded the data for small system sizes $L \leqslant 8$ and for small distances $y \leqslant L / 4$. Then, the fit yields $X_{t 1}=0.198(3)$, in good agreement with the theoretical value $X_{t 1}=1 / 5$.

\section{Special phase transitions}

As for the case of the tricritical $q=1$ Potts model, we expect that the geometric effect in the tricritical BC model can be asymptotically compensated by the enhancements of surface parameters $K_{s}$ and $D_{s}$. To test this expectation, we used a combination of the Wolff and Metropolis methods to simulate the BC model on open cylinders with size $L \times L$. The simulations were performed at the bulk tricritical point mentioned above, and we took the surface parameters as $\kappa_{d}=0$ and $H_{s}=0$. The system sizes assumed 14 odd values in the range $9 \leqslant L \leqslant 121$, and we sampled the magnetization density and the vacancy density $\rho$ for several values of $\kappa_{k}$. Further, we defined two dimensionless ratios as

$$
Q_{b}=\left\langle m_{b}^{2}\right\rangle^{2} /\left\langle m_{b}^{4}\right\rangle \text { and } Q_{s}=\left\langle m_{s 1} m_{s 2}\right\rangle^{2} /\left\langle\left(m_{s 1} m_{s 2}\right)^{2}\right\rangle,
$$

where $m_{b}, m_{s 1}$, and $m_{s 2}$ are the magnetization densities on the lines $y=(L+1) / 2, y=1$, and $y=L$, respectively. These di-

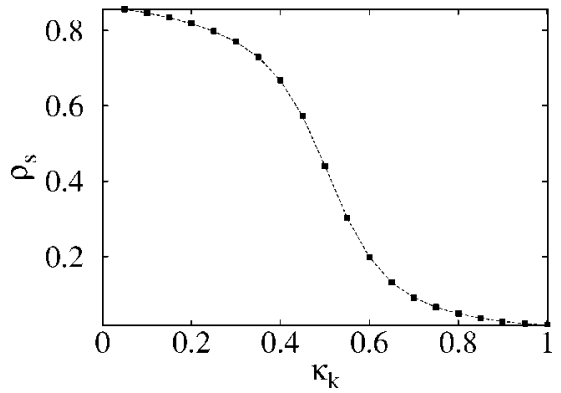

FIG. 7. Edge vacancy density $\rho_{s}$ of the tricritical BC model vs coupling enhancement $\kappa_{k}$. The other surface parameters are $\kappa_{d}=0$ and $H_{s}=0$, and the system size is $L=15$.

mensionless quantities are closely related to the Binder ratio, and they are useful in Monte Carlo analyses of critical points, because their asymptotic values at criticality are universal.

The absolute value of the surface magnetization $\left|m_{s}\right|$ and the edge vacancy density $\rho_{s}$ for system size $L=15$ are shown in Figs. 6 and 7, respectively. These figures illustrate that, for coupling enhancements $\kappa_{k}>0.6$, the open edges are dominated by Ising spins so that spontaneous order occurs on the one-dimensional edges. Further, the clean intersection of the $Q_{b}$ data in Fig. 8 reveals a second-order phase transition near $\kappa_{k}=0.56$. We fitted the data of $Q_{b}$ and $Q_{s}$ by the formula

$$
\begin{aligned}
Q\left(\kappa_{k}, L\right)= & Q_{c}+\sum_{k=1}^{4} a_{k}\left(\kappa_{k}-\kappa_{k c}\right)^{k} L^{k y_{t s}^{(\mathrm{s})}}+b_{1} L^{y_{1}}+b_{2} L^{y_{2}}+b_{3} L^{y_{3}} \\
& +b_{4} L^{y_{4}}+c\left(\kappa-\kappa_{k c}\right) L^{y_{t s}^{(\mathrm{s})}+y_{1}}+n\left(\kappa-\kappa_{k c}\right)^{2} L^{y_{t s}^{(\mathrm{s})}}, \quad(17)
\end{aligned}
$$

where the terms with $b_{1}, b_{2}$, and $b_{3}$ account for finite-size corrections. The exponent $y_{1}=y_{i}=-1$ arises from the leading irrelevant thermal scaling field [20,21]. More generally, we expect analytic finite-size corrections with exponents $y_{j}=$ $-n$ with integer $n \geqslant 1$. Thus, the exponents $y_{2}, y_{3}$, and $y_{4}$ were taken as $-2,-3$, and -4 , respectively. The term with $c$ describes the "mixed" effect of the relevant and the irrelevant thermal scaling fields, and the last term in Eq. (17) is due to the fact that the surface thermal scaling field can be a

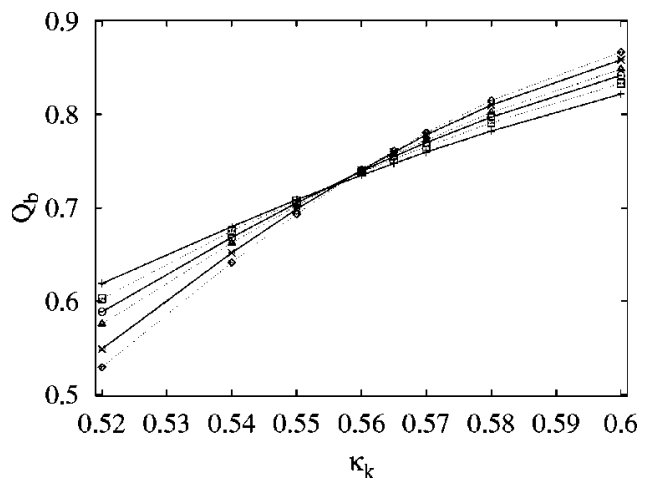

FIG. 8. Bulk magnetic ratio $Q_{b}$ of the tricritical BC model vs coupling enhancement $\kappa_{k}$. The other surface parameters are $\kappa_{d}=0$ and $H_{s}=0$, and the system sizes are $L=11(+), 15(\square), 19(\bigcirc), 23$ $(\triangle), 31(\times)$, and $39(\diamond)$. 


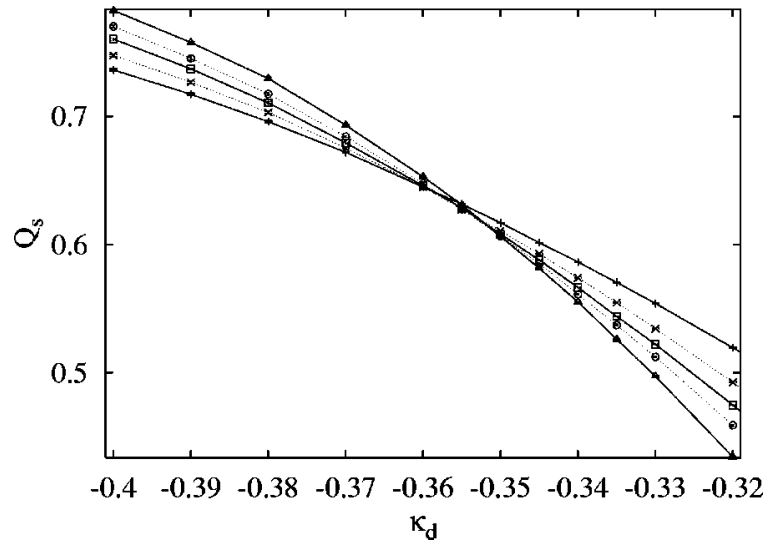

FIG. 9. Surface magnetic ratio $Q_{s}$ of the tricritical BC model vs chemical-potential enhancement $\kappa_{d}$. The other surface parameters are $\kappa_{k}=0$ and $H_{s}=0$, and the system sizes are $L=9(+), 13(\times), 17$ $(\square), 21(\bigcirc)$, and $29(\triangle)$.

nonlinear function of $\kappa_{k}$. After a cutoff for small system sizes $L \leqslant 11$, the fit of $Q_{b}$ yields $Q_{b c}=0.765(4), \kappa_{k c}=0.5660(4)$, and $y_{t s}^{(\mathrm{s})}=0.407(8)$, and the fit of $Q_{s}$ yields $Q_{s c}=0.566(3)$, $\kappa_{k c}=0.5664(4)$, and $y_{t s}^{(\mathrm{s})}=0.395(7)$. These two fits are consistent with each other, and the results for $y_{t s}^{(\mathrm{s})}$ are equal to the exact value $2 / 5[23,24]$ within the estimated error margins.

Near the above estimated special transition $\kappa_{k}=\kappa_{k c}$ and $\kappa_{d}=0$, the surface magnetic susceptibility $\chi_{s}=L\left\langle m_{s}^{2}\right\rangle$ was sampled, and the Monte Carlo data were fitted by

$$
\begin{aligned}
\chi_{s}\left(\kappa_{k}, L\right)= & r_{0}+r_{1}\left(\kappa-\kappa_{k c}\right)+r_{2}\left(\kappa-\kappa_{k c}\right)^{2}+L^{2 y_{h s}^{(\mathrm{s})}-1} \\
& \times\left[\sum_{k=0}^{4} a_{k}\left(\kappa-\kappa_{k c}\right)^{k} L^{k y_{t s}^{(\mathrm{s})}}+b_{1} L^{y_{1}}+b_{2} L^{y_{2}}+b_{3} L^{y_{3}}\right. \\
& \left.+c\left(\kappa-\kappa_{k c}\right) L^{y_{t s}^{(\mathrm{s})}+y_{1}}+n\left(\kappa-\kappa_{k c}\right)^{2} L^{y_{t s}^{(\mathrm{s})}}\right]
\end{aligned}
$$

The terms with $r_{0}, r_{1}$, and $r_{2}$ come from differentiations of the analytical part of the free energy with respect to the surface magnetic scaling field. We fixed the surface thermal exponent $y_{t s}^{(\mathrm{s})}$ at the value $2 / 5[23,24]$, and obtained $\kappa_{k c}$

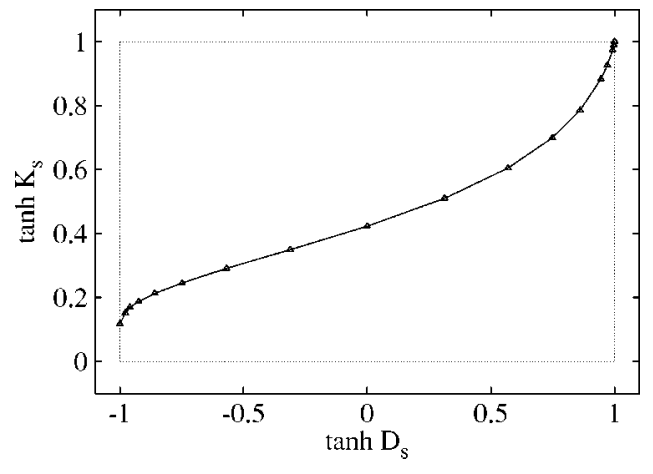

FIG. 10. Line of special transitions of the tricritical BC model in the parameter space $\left(\tanh K_{s}, \tanh D_{s}\right)$. The symbols $\triangle$ represent the numerical data. When the bulk coupling $K$ is varied, the transition on the edge is first order in the region above the curve.

$=0.5658(8)$ and $y_{h s}^{(\mathrm{s})}=0.914(8)$, where the error margins are quoted as two standard deviations. The corresponding scaling dimension $X_{h s}^{(\mathrm{s})}=1-y_{h s}^{(\mathrm{s})}=0.086(8)$ is marginally consistent with the bulk magnetic scaling dimension $X_{h}=3 / 40$ but also with the exact value $1 / 10[23,24]$. We shall come back to this point in Sec. V.

Just like $\kappa_{k}$, the enhancement $\kappa_{d}$ of the surface chemical potential also induces a "special transition." This is illustrated by the $Q_{s}$ data in Fig. 9 for the case $\kappa_{k}=0$. The fit of the $Q_{s}$ data by Eq. (17) yields a critical point at $\kappa_{d c}=$ $-0.344(2)$. Using the same technique, we have determined a number of special critical points in the parameter space $\left(K_{s}, D_{s}\right)$, which are listed in Table I. On this basis, the line of special edge transitions is shown in Fig. 10. For the limit $K_{s} \rightarrow \infty$, the edge transition is first order, and separates a state with edges fully occupied by the vacancies from one with fully magnetized edges. From the relative statistical weights of these phases, the transition is simply obtained as $K_{s c} / D_{s c}=1$. For the opposite limit $D_{s} \rightarrow-\infty$, no vacancies occur on the edges. We simulated this limit for system sizes in the range $11 \leqslant L \leqslant 111$. We still find a second-order transition at $K_{s c}=K_{t}\left(1+\kappa_{k c}\right)=0.1183(8)$. The surface critical coupling strength $K_{s c}$ is quite small in comparison with the bulk tricritical value $K_{t}=1.6431759(1)$. Near the critical point $K_{s c}$, the data of the surface susceptibility $\chi_{s}$ were fitted by

TABLE I. Numerical results for several special edge transition points of the tricritical BC model in the space $\left(K_{s}, D_{s}\right)$. The critical values are given in terms of the surface enhancements $\kappa_{k c}$ and $\kappa_{d c}$; the corresponding values of $K_{s c}$ and $D_{s c}$ are $K_{s c}=K_{t}\left(1+\kappa_{k c}\right)$ and $K_{d c}=D_{t}\left(1+\kappa_{d c}\right)$.

\begin{tabular}{llllll}
\hline \hline$\kappa_{d c}$ & $-\infty$ & -1.7 & -1.6 & -1.5 & -1.4 \\
$\kappa_{k c}$ & $-0.9280(4)$ & $-0.9050(4)$ & $-0.8964(4)$ & $-0.8845(4)$ & $-0.8686(4)$ \\
$\kappa_{d c}$ & -1.3 & -1.2 & -1.1 & -1.0 & -0.9 \\
$\kappa_{k c}$ & $-0.8474(4)$ & $-0.8175(4)$ & $-0.7778(7)$ & $-0.7252(7)$ & $-0.6576(7)$ \\
$\kappa_{d c}$ & -0.8 & -0.7 & -0.6 & -0.45 & -0.35 \\
$\kappa_{k c}$ & $-0.5737(8)$ & $-0.4732(8)$ & $-0.3563(9)$ & $-0.1261(8)$ & $-0.0095(9)$ \\
$\kappa_{d c}$ & -0.15 & 0 & 0.6 & & \\
$\kappa_{k c}$ & $0.3096(8)$ & $0.5662(4)$ & $1.6665(8)$ & & \\
\hline \hline
\end{tabular}




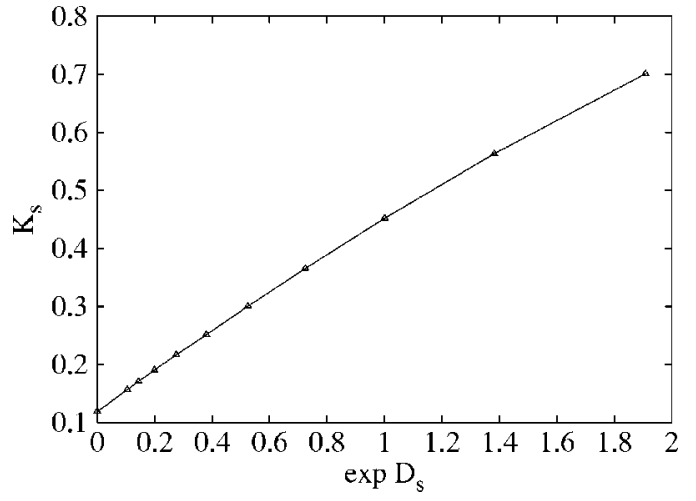

FIG. 11. Line of special transitions of the tricritical BC model in the parameter space $\left(K_{s}, e^{D_{s}}\right)$.

Eq. (18). We obtain the surface magnetic exponent as $y_{h s}^{(\mathrm{s})}$ $=0.098(2)$, in good agreement with the exact value $1 / 10$ $[23,24]$.

As indicated in Fig. 10, the line of the special transitions exhibits two bends near the limits $D_{s} \rightarrow \pm \infty$. To display its behavior for large $D_{s} \rightarrow-\infty$ in more detail, this line is shown again in Fig. 11 in the parameter space $\left(K_{s}, e^{D_{s}}\right)$.

\section{Extraordinary phase transitions}

In the upper region of the critical line in Fig. 10, the edges and the bulk undergo a first- and a second-order transition, respectively, when the bulk coupling $K$ is varied, i.e., the surface magnetization density $m_{s}$ displays a discontinuity. In comparison with the three-dimensional Ising model, this can be considered to correspond with the coincidence of the "surface" and the "extraordinary" transitions. The existence of spontaneous edge order is only possible because the bulk is tricritical. At this point, spins on the edges in effect interact via sufficiently long-ranged bulk correlations, so that the edge correlations also become long ranged.

It seems reasonable to expect that the bulk critical properties are reflected on the edges even if the edge transition is first order. As a test, we simulated the tricritical BC model for $\kappa_{d}=0$ and $\kappa_{k}>0.6$. The system sizes were taken in the range $9 \leqslant L \leqslant 185$, and part of the $Q_{b}$ data are shown in Fig. 12. The clean intersection in Fig. 12 indicates a fixed point

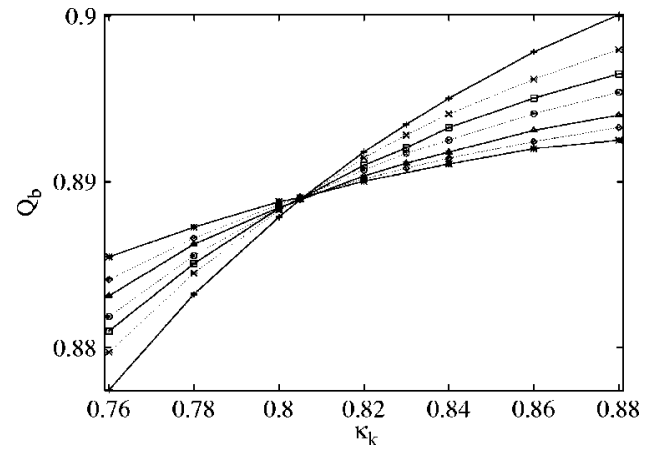

FIG. 12. Bulk magnetization moment ratio $Q_{b}$ of the tricritical BC model vs surface coupling enhancement $\kappa_{k}$. The other surface parameters are $\kappa_{d}=0$ and $H_{s}=0$. The data points $+, \times, \square, \bigcirc, \triangle$, $\diamond$, and $*$ represent $L=7,9,11,13,17,21$, and 29 , respectively. The clean intersection of these data lines implies that, in addition to the "special" transition $\kappa_{k}=\kappa_{k c}=0.5662(5)$, there is another "fixed" point at $\kappa_{k} \approx 0.81$. However, in contrast to Fig. 8, the slope of these lines is a decreasing function of the system size $L$. This means that this fixed point is stable in the $\kappa_{k}$ direction.

near $\kappa_{k}=0.81$. The decreasing slope as a function of $L$ indicates that this fixed point is stable in the $\kappa_{k}$ direction. Naturally, the question arises what critical exponent governs the renormalization flow in the $\kappa_{k}$ direction. For this purpose, we fitted the $Q_{b}$ data by Eq. (17), where the exponent $y_{t s}^{(\mathrm{s})}$ is replaced by $y_{t s}^{(\mathrm{e})}$. After discarding data for small system sizes $L \leqslant 9$, we obtain $Q_{c}=0.892(2)$ and $y_{t s}^{(\mathrm{e})}=-0.80(5)$. We note that, for an arbitrary $d$-dimensional system, a surface thermal exponent $y_{t s}=-1$ has been reported [35] to occur. However, the result $y_{t s}^{(\mathrm{e})}=-0.80(5)$ is slightly different from this exact value, which dominates the range of the ordinary transitions.

For a further illustration of the edge critical properties in the range of the extraordinary transition, we fitted the $m_{s}^{2}$ data at $\kappa_{k}=0.805$, which are listed in Table II, by the formula

$$
m_{s}^{2}=a_{0}^{2}+L^{-2 X_{h s}^{(\mathrm{e})}}\left(b_{0}+b_{1} L^{y_{t s}^{(\mathrm{e})}}+b_{2} L^{y_{i}}+b_{3} L^{-2}+b_{4} L^{-3}\right),
$$

where the term $a_{0}$ represents the spontaneous edge magnetization density. The exponent $y_{t s}^{(\mathrm{e})}$ was fixed at the estimated

TABLE II. Monte Carlo data for the second moment $m_{s}^{2}$ of the surface magnetization density at the extraordinary transition in the tricritical $\mathrm{BC}$ model. The surface parameters are $H_{s}=0, \kappa_{d}=0$, and $\kappa_{k}$ $=0.805$.

\begin{tabular}{llllll}
\hline \hline$L$ & 9 & 11 & 13 & 15 & 17 \\
$m_{s}^{2}$ & $0.91729(1)$ & $0.91560(1)$ & $0.91399(1)$ & $0.91262(1)$ & $0.91145(1)$ \\
$L$ & 19 & 21 & 23 & 25 & 29 \\
$m_{s}^{2}$ & $0.91044(1)$ & $0.90960(1)$ & $0.90886(1)$ & $0.90821(1)$ & $0.90717(1)$ \\
$L$ & 33 & 37 & 45 & 55 & 65 \\
$m_{s}^{2}$ & $0.90631(1)$ & $0.90562(1)$ & $0.90459(1)$ & $0.90367(1)$ & $0.90299(1)$ \\
$L$ & 85 & 105 & 145 & 185 & \\
$m_{s}^{2}$ & $0.90206(1)$ & $0.90151(1)$ & $0.90076(1)$ & $0.90031(1)$ & \\
\hline \hline
\end{tabular}




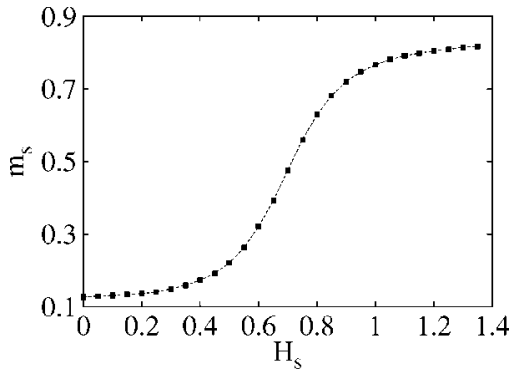

FIG. 13. Edge magnetization density $m_{s}$ of the tricritical BC model vs surface magnetic field $H_{s}$ The system size is $L=32$, and surface parameters are $\kappa_{d}=0$ and $\kappa_{k}=0$.

value $-0.80(5)$, and we obtain $a_{0}=0.94776(4)$ and $X_{h s}^{(\mathrm{e})}$ $=0.3987(15) \approx 2 / 5$. Thus, critical correlations still occur on the edges.

\section{Field-driven edge transition}

It seems reasonable that, in analogy with the enhancements of the surface couplings $K_{s}$ and the chemical potential $D_{s}$, the geometric effect can also be compensated by the surface magnetic field $H_{s}$. Thus, we simulated the tricritical BC model for surface parameters $\kappa_{d}=\kappa_{k}=0$ but $H_{s} \neq 0$. The edge magnetization density $m_{s}$ is shown versus $H_{s}$ in Fig. 13 for system size $L=32$. It behaves consistently with the above expectation. To test for the presence of a field-driven edge phase transition, we defined the ratio $Q_{s f}=\left\langle\left(m_{s}\right.\right.$ $\left.\left.-\left\langle m_{s}\right\rangle\right)^{2}\right\rangle^{2} /\left\langle\left(m_{s}-\left\langle m_{s}\right\rangle\right)^{4}\right\rangle$. The $Q_{s f}$ data in the range $8 \leqslant L$ $\leqslant 48$ were fitted by Eq. (17), and we obtained $Q_{s f c}$ $=0.4419(10), \quad H_{s c}=0.6772(10)$, and $y_{h s}^{\mathrm{f}}=0.405(10) \approx 2 / 5$. This result, in particular the relevant exponent $y_{h s}^{\mathrm{f}}$, confirms the existence of the phase transition.

The phase diagram in the parameter space $\left(\kappa_{k}, H_{s}\right)$ is sketched in Fig. 14 for $\kappa_{d}=0$. The numerical results for the estimated critical points, as denoted by $\times$ in Fig. 14, are listed in Table III. It is clear from Fig. 14 that the special transition, at $\kappa_{k c}=0.5662(4), H_{s}=0$, behaves as a "multicritical" point, because several phase transition lines merge in this point.

\section{Bulk first-order range: $K>K_{t}$}

For $K>K_{t}$, the bulk of the BC model exhibits a line of first-order transitions, which separates the ferromagnetic

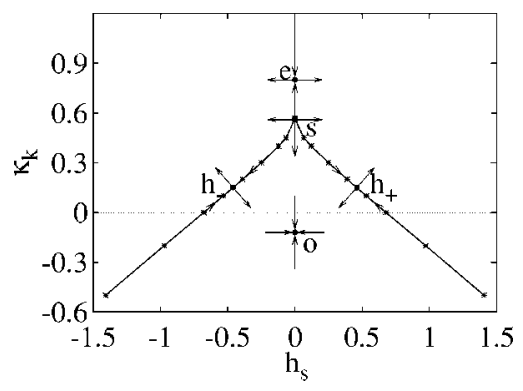

FIG. 14. Sketch of the phase transitions of the tricritical BC model in the parameter space $\left(\kappa_{k}, H_{s}\right)$ with $\kappa_{d}=0$. The fixed points are denoted by the black circles, and the arrows show the direction of the renormalization flow.
TABLE III. Numerical determinations of several field-driven edge transitions for the tricritical BC model in the parameter space $\left(\kappa_{k}, H_{s}\right)$. The surface chemical-potential enhancement is $\kappa_{d}=0$.

\begin{tabular}{lllll}
\hline \hline$\kappa_{k c}$ & 0.45 & 0.4 & 0.3 & 0.2 \\
$H_{s c}$ & $0.0661(10)$ & $0.1212(8)$ & $0.2500(10)$ & $0.3880(8)$ \\
$\kappa_{k c}$ & 0.1 & 0 & -0.2 & -0.5 \\
$H_{s c}$ & $0.5315(10)$ & $0.6772(10)$ & $0.9720(10)$ & $1.4080(10)$ \\
\hline \hline
\end{tabular}

phases from the vacancy-dominated phase. On this transition line, just as for the tricritical $q=1$ Potts model, the surface parameters can also induce first-order transitions. These transitions occur simultaneously on the edges and in the bulk. As an example, we simulated the BC model for $K=1.8$ and $D$ $=3.55535$, which is very close to the line of the bulk transitions. The surface parameters were fixed at $\kappa_{d}=H_{s}=0$, and we sampled the bulk and edge vacancy densities $\rho_{b}$ and $\rho_{s}$ on the lines $y=(L+1) / 2$ and $y=1, L$, respectively. The hysteresis loops of $\rho_{b}$ and $\rho_{s}$ for system size $L=63$ are shown in Figs. 15 and 16, respectively. The hysteresis loop of $\rho_{s}$ is rather asymmetric.

According to the investigations in this section, the edge phase transitions of the Blume-Capel model are sketched in Fig. 17. The shaded area represents the surface of bulk phase transitions. For $K<K_{t}$, only the ordinary transitions occur on the edges, so that all renormalization flow lines in this part of the critical surface end in a single fixed point. Along the bulk tricritical line $K=K_{t}$ and $D=D_{t}$, there are three fixed points, representing the ordinary, the special, and the extraordinary phase transitions, respectively. For $K>K_{t}$, there is a line of first-order transitions, which is denoted as the dashed line in Fig. 17. On the left- and the right-hand sides of this line, the edges and the bulk of the BC model are dominated by the vacancies and the Ising spins, respectively.

\section{TRICRITICAL THREE-STATE POTTS MODEL}

The Hamiltonian of the dilute $q=3$ Potts model in the torus geometry is described by Eq. (5) with $q=3$. The bulk phase diagram of this model is analogous to that of the Blume-Capel model described by Eq. (12). At tricriticality,

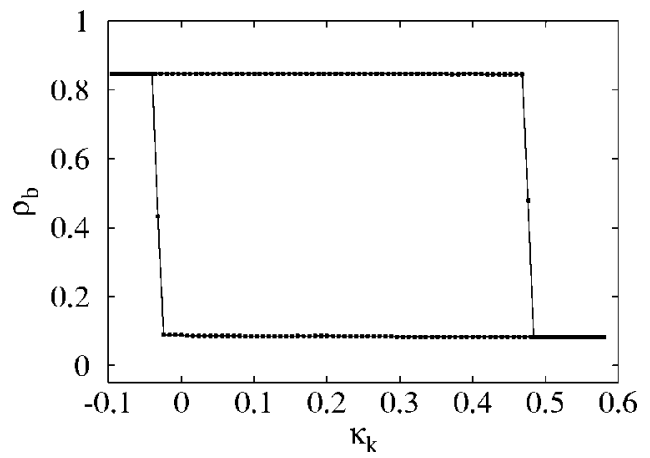

FIG. 15. Hysteresis loop of the bulk vacancy density $\rho_{b}$ of the BC model with $K=1.8$ and $D=3.55535$ vs surface coupling enhancement $\kappa_{k}$. The system size is $L=63$. 


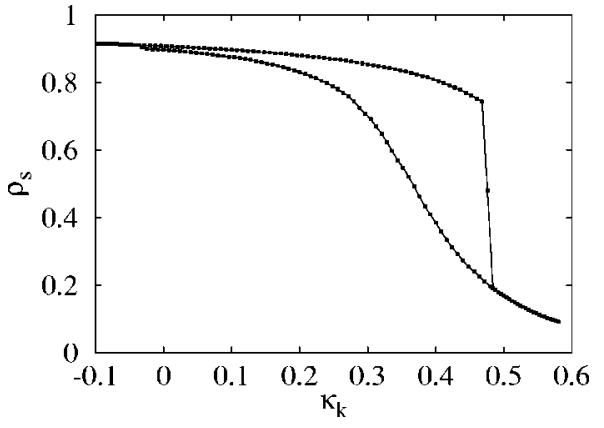

FIG. 16. Hysteresis loop of the edge vacancy density $\rho_{s}$ of the BC model with $K=1.8$ and $D=3.55535$ vs surface coupling enhancement $\kappa_{k}$. The system size is $L=63$.

the leading and subleading bulk thermal exponents are $y_{t 1}$ $=12 / 7$ and $y_{t 2}=4 / 7$, respectively, and the magnetic ones are $y_{h 1}=40 / 21$ and $y_{h 2}=22 / 21[20,21]$. By means of a sparse transfer-matrix technique, the tricritical point on the square lattice has been determined [33] as $K_{t}=1.649913(5)$ and $D_{t}=3.152173(10)$; the tricritical vacancy density is $\rho_{t}$ $=0.34572(5)$.

On the $L \times L$ open cylinder, the Hamiltonian of the dilute $q=3$ Potts model reads

$$
\begin{aligned}
\mathcal{H}_{\mathrm{O}} / k_{\mathrm{B}} T-\mathcal{H}_{\mathrm{P}} / k_{\mathrm{B}} T \\
=K \sum_{x=1}^{L} \delta_{\sigma_{x, 1}, \sigma_{x, L}}\left(1-\delta_{\sigma_{x, 1}, 0}\right)-K \kappa_{k} \sum_{x=1}^{L}\left[\delta_{\sigma_{x, 1}, \sigma_{x+1,1}}\right. \\
\left.\quad \times\left(1-\delta_{\sigma_{x, 1}, 0}\right)+\delta_{\sigma_{x, L}, \sigma_{x+1, L}}\left(1-\delta_{\sigma_{x, L}, 0}\right)\right] \\
\quad-D \kappa_{d} \sum_{x=1}^{L}\left(\delta_{\sigma_{x, 1}, 0}+\delta_{\sigma_{x, L}, 0}\right)-H_{s 1} \sum_{k=1}^{L}\left(\delta_{\sigma_{x, 1}, 1}+\delta_{\sigma_{x, L}, 1}\right) \\
\quad+\frac{H_{s 1}}{2} \sum_{k=1}^{L}\left(\delta_{\sigma_{x, 1}, 2}+\delta_{\sigma_{x, L}, 2}\right)+\frac{H_{s 1}}{2} \sum_{k=1}^{L}\left(\delta_{\sigma_{x, 1}, 3}+\delta_{\sigma_{x, L}, 3}\right) .
\end{aligned}
$$

The surface magnetic field $H_{s 1}$ serves to enhance the statistical weight of the Potts state $\sigma=1$ with respect to states $\sigma$ $=2$ and $\sigma=3$.

In analogy with the BC model, the system (20) has a line of bulk critical points for $K<K_{t}$, in the same universality class as the "pure" $q=3$ Potts model. Thus, only the ordinary phase transition occurs on the open edges, with a surface magnetic exponent $y_{h s}^{(\mathrm{o})}=1 / 3$, as predicted by Eq. (4). For $K>K_{t}$, a first-order transition can be induced by enhancements of the surface couplings and the chemical potential. In the present work, we concentrate on the case that the bulk is precisely at the tricritical point.

\section{A. Special phase transitions}

We simulated the tricritical $q=3$ Potts model (20) on the $L \times L$ open cylinder by means of a combination of the Metropolis and Wolff methods, with the linear size in the range $7 \leqslant L \leqslant 65$. The bulk parameters were set at the aforementioned tricritical point, and the surface parameters at $H_{s 1}=0$

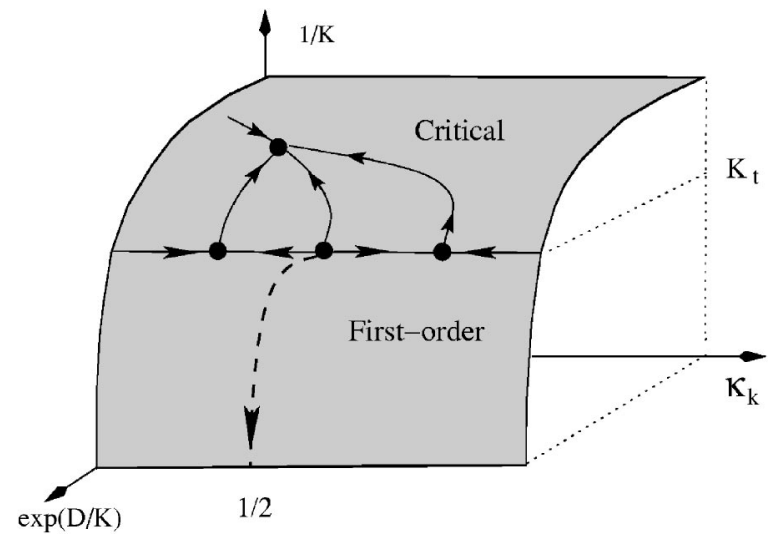

FIG. 17. Sketch of the edge phase diagram of the BC model for $H_{s}=0$. The fixed points are shown as black circles, and the arrows show the renormalization flow.

and $\kappa_{d}=0$. The edge order parameter was defined as $m_{s}^{2}$ $=\left[\left(\rho_{1}-\rho_{2}\right)^{2}+\left(\rho_{2}-\rho_{3}\right)^{2}+\left(\rho_{3}-\rho_{1}\right)^{2}\right] / 2$, in which $\rho_{i}$ is the density of the edge spins in state $i$. Accordingly, we sampled the ratio $Q_{s 1}=\left\langle m_{s}^{2}\right\rangle^{2} /\left\langle m_{s}^{4}\right\rangle$. The $Q_{s 1}$ data are partly shown in Fig. 18. They indicate a special edge transition near $\kappa_{k}=0.7$. The $Q_{s 1}$ data were fitted by Eq. (17), in which the correction exponents were fixed at $y_{1}=y_{i}=-10 / 7$ [3], $y_{2}=-1, y_{3}=-2$, and $y_{4}=-3$. We obtain $Q_{s 1 c}=0.941(2), \kappa_{k c}=0.702(2)$, and $y_{t s}^{(\mathrm{s})}=0.282(5)$. Near this special phase transition, i.e., $\kappa_{k c}$ $=0.702(2)$ and $\kappa_{d}=0$, we also analyzed the edge susceptibility $\chi_{s}$ by Eq. (18). The fit yields $X_{h s}^{(\mathrm{s})}=0.133(15)$.

\section{B. Field-driven edge transitions}

Next, we simulated the tricritical $q=3$ Potts model (20) in the presence of the surface magnetic field $H_{s 1}$; the other surface enhancements were taken as $\kappa_{k}=\kappa_{d}=0$. The system sizes were taken as ten odd values in the range $7 \leqslant L \leqslant 49$, and we sampled the bulk ratio $Q_{b}$ in Eq. (16). Analogous to the case of the tricritical $\mathrm{BC}$ model, edge phase transitions are introduced by the field $H_{s 1}$. Nevertheless, the symmetry between the positive and the negative field is now absent. The $Q_{b}$ data were fitted by Eq. (17), and we found two edge

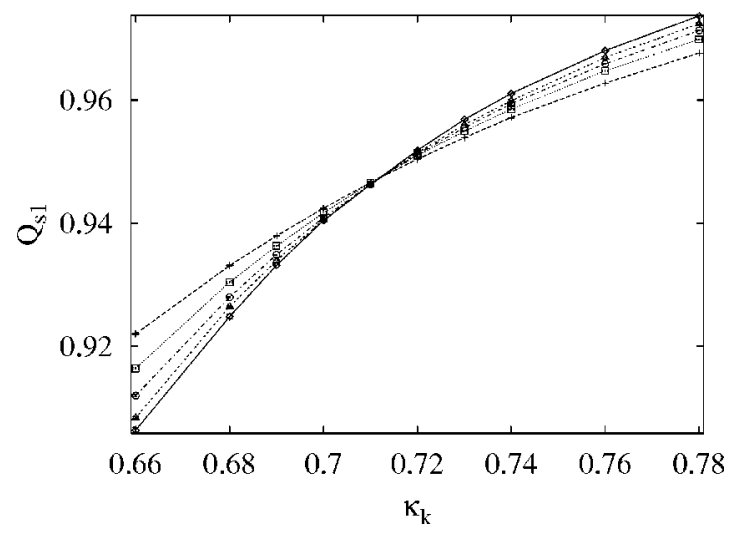

FIG. 18. Surface magnetic ratio $Q_{s 1}$ of the tricritical $q=3$ model vs coupling enhancement $\kappa_{k}$. The data points $+, \square, \bigcirc, \triangle$, and $\diamond$ represent $L=7,15,23,31$, and 39 , respectively. 


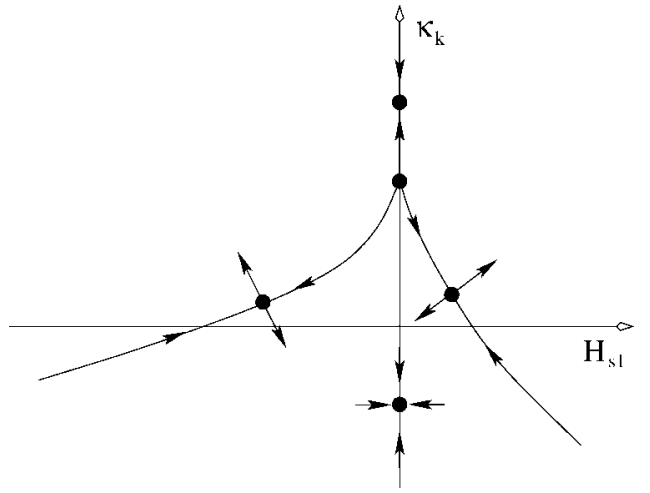

FIG. 19. Sketch of the edge phase diagram of the tricritical $q$ $=3$ Potts model in the $\left(H_{s 1}, \kappa_{k}\right)$ plane for $\kappa_{d}=0$. The fixed points are denoted by black circles, and the arrows show the renormalization flow.

transitions at $H_{s 1 c}=0.5710(15)$ and $-2.27(3)$. At these two points, the asymptotic values of the ratio $Q_{b}$ are $0.462(4)$ and $0.232(8)$, respectively; those of the renormalization exponent $y_{h s}^{\mathrm{f}}$ are $0.278(8)$ and $0.280(8)$, respectively, which are identical to each other within the estimated error margins. From these results, we conjecture the loci of the edge transitions in the plane $\left(\kappa_{k}, H_{s 1}\right)$ as sketched in Fig. 19.

In addition to $H_{s 1}$, the surface magnetic fields, $H_{s 2}$ and $H_{s 3}$, can also be applied to the Potts system (20). Thus, for the case $\kappa_{d}=\kappa_{k}=0$, Fig. 20 illustrates the edge phase diagram in the space $\left(H_{s 1}, H_{s 1}, H_{s 3}\right)$.

\section{DISCUSSION}

By means of Monte Carlo simulations and finite-size scaling, we have found that rich surface critical phenomena still occur in two-dimensional systems with short-range interactions only. In particular, when the bulk of a Potts model is at the tricritical point, edge transitions are introduced by enhancements of the surface couplings and the chemical potential, and by a surface magnetic field. For the critical branch of the Potts model, however, only the ordinary phase transition exists on the one-dimensional edges. Thus, one con-

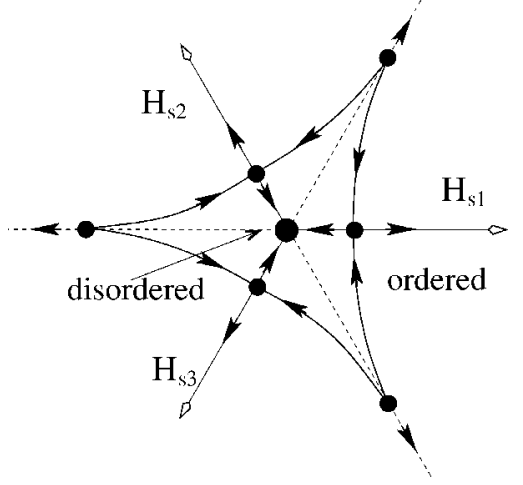

FIG. 20. Sketch of the edge phase diagram of the tricritical $q$ $=3$ Potts model as a function the surface fields for $\kappa_{d}=\kappa_{k}=0$. The fixed points are denoted by black circles, and the arrows illustrate the renormalization flows.
TABLE IV. Theoretical predictions of the surface thermal and magnetic exponents $y_{t s}^{(\mathrm{s})}$ and $y_{h s}^{(\mathrm{s})}$ at the special phase transitions for the tricritical $q$-state Potts model with $q=1,2,3$, and 4 . The parameter $g$ is the Coulomb gas coupling constant.

\begin{tabular}{llllll}
\hline \hline Model & $q=0$ & $q=1$ & $q=2$ & $q=3$ & $q=4$ \\
\hline$g$ & 6 & $16 / 3$ & 5 & $14 / 3$ & 4 \\
$y_{t s}^{(\mathrm{s})}$ & $2 / 3$ & $1 / 2$ & $2 / 5$ & $2 / 7$ & 0 \\
$y_{h s}^{(\mathrm{s})}$ & 1 & $15 / 16$ & $9 / 10$ & $6 / 7$ & $3 / 4$ \\
\hline
\end{tabular}

cludes that, in two dimensions, it depends on the strength of bulk critical fluctuations whether other types of edge phase transitions can occur.

Let us now address the question of what are the exact values of the critical exponents describing the edge phase transitions described in this work. At the special transitions, one has the exact result $y_{t s}^{(\mathrm{s})}(q=1)=1 / 2$ for the tricritical $q$ $=1$ Potts model. For the case of the tricritical Blume-Capel model, in the context of superconformal field theory, it has been predicted that the surface thermal and magnetic exponents are $[23,24] y_{t s}^{(\mathrm{s})}=2 / 5$ and $y_{h s}^{(\mathrm{s})}=9 / 10$, respectively. For the whole tricritical branch of the Potts model, it has been recently conjectured [28] that, in terms of the Coulomb gas coupling constant $g$, the exact expressions of $y_{h s}^{(\mathrm{s})}$ and $y_{t s}^{(\mathrm{s})}$ read

$$
y_{h s}^{(\mathrm{s})}=(3 g-6) / 2 g \text { and } y_{t s}^{(\mathrm{s})}=2-8 / g,
$$

respectively. These values of $y_{h s}^{(\mathrm{s})}$ and $y_{t s}^{(\mathrm{s})}$ are one-half of the leading and subleading bulk thermal exponents, $y_{t 1}$ and $y_{t 2}$, respectively. For the tricritical Potts models with $q=0,1,2$, 3 , and 4, the results according to Eq. (21) are listed in Table IV. A remarkable feature is that the expression of $y_{t s}^{(\mathrm{s})}$ in Eq. (21) can be simply obtained by substituting $g=16 / g^{\prime}$ in Eq. (4), which describes the surface magnetic exponent $y_{h s}^{(\mathrm{o})}$ at the ordinary phase transitions. The underlying meaning of this procedure is clear for the tricritical $q=1$ Potts model, because the special transition of this model is just the ordinary transition of the Ising model and the Coulomb gas coupling of these two models are $g=16 / 3$ and 3, respectively. For a pair of critical and tricritical Potts models, we note that the relation $g g^{\prime}=16$ has been reported [36,37] in other cases. Thus, Eq. (21) might mean that the effect of surface couplings $K_{s}$ in a tricritical Potts model is equivalent to that of the magnetic field $H_{s}$ in a critical system, the two models being related as $g g^{\prime}=16$.

For the tricritical $q=1$ Potts model, Eq. (21) predicts a surface magnetic exponent $y_{h s}^{(\mathrm{s})}=15 / 16$. It is known that the bulk Potts magnetic scaling dimension $X_{h}=5 / 96$ is the fractal dimension of Ising clusters, which connect nearestneighbor Ising spins of the same sign. Thus, one would expect that the exponent $y_{h s}^{(\mathrm{s})}$ governs the scaling behavior of the correlation function $g^{(\mathrm{II})}(r)$, which is defined as the probability that a pair of edge points is in the same Ising cluster. However, it has been shown that the decay of $g^{(\mathrm{I})}(r)$ is described by a geometric scaling dimension $X_{p}=1 / 6$ [28], dif- 
ferent from $X_{h s}^{(\mathrm{s})}=1 / 16$. The physical interpretation of $y_{h s}^{(\mathrm{s})}$ is so far not clear.

For the tricritical BC model, the results obtained from Eq. (21) are in agreement with the predictions in Refs. [23,24]. In particular, the prediction $y_{t s}^{(\mathrm{s})}=2 / 5$ is well confirmed by our numerical result $y_{t s}^{(\mathrm{s})}=0.395(7)$. However, the exact value $y_{h s}^{(\mathrm{s})}=9 / 10$ is only marginally consistent with the result $y_{h s}^{(\mathrm{s})}$ $=0.914(8)$ for the case $\kappa_{d}=H_{s}=0$ and $\kappa_{k c}=0.5662(4)$. On the other hand, at the critical point $\kappa_{k c}=-0.9280(4), \kappa_{d}=-\infty$, the result $y_{h s}^{(\mathrm{s})}=0.902(2)$ is in $\operatorname{good}$ agreement with $y_{h s}^{(\mathrm{s})}=9 / 10$. This might be taken as a suggestion that the end point of the line of special transitions at $\kappa_{d}=-\infty$ in Fig. 10 acts as an unstable fixed point with the expected exponent $y_{h s}^{(\mathrm{s})}=9 / 10$, while the rest of the line is attracted by another fixed point. However, the numerical evidence is only marginal, and moreover, if the end point at $\kappa_{d}=-\infty$ is a fixed point, then one would in general expect that the line of special transitions, when parametrized by the activity of the vacancies and the surface coupling enhancement, displays a singularity at $\kappa_{d}=-\infty$. No sign of such a singularity is visible in Fig. 11.

For the tricritical $q=3$ model, the predictions in Table IV are consistent with the numerical determinations $y_{t s}^{(\mathrm{s})}$ $=0.282(5)$ and $y_{h s}^{(s)}=0.867(15)$.
The $q=4$ Potts model is a marginal case for several reasons. First, the critical and the tricritical branches of the Potts model join at $q=4$. Second, Eq. (4) predicts that, at the ordinary phase transitions, the surface magnetic scaling field is marginal, i.e., $y_{h s}^{(\mathrm{o})}=0$. Third, Eq. (21) yields $y_{t s}^{(\mathrm{s})}=0$, so that the surface coupling and the chemical-potential enhancement become marginal. However, the prediction $y_{h s}^{(\mathrm{s})}=3 / 4$ according to Eq. (21) is apparently different from the exponent $y_{h s}^{(\mathrm{o})}=0$. The result $y_{t s}^{(\mathrm{s})}=0$ also differs from the existing surface thermal exponent $y_{t s}=-1$ [35]. These phenomena indicate that there exists a special phase transition for the $q=4$ Potts model.

\section{ACKNOWLEDGMENTS}

The authors are indebted to Dr. J. R. Heringa and X. F. Qian for valuable discussions. This research was supported by the Dutch FOM foundation ("Stichting voor Fundamenteel Onderzoek der Materie") which is financially supported by the NWO ("Nederlandse Organisatie voor Wetenschappelijk Onderzoek").
[1] K. Binder, in Phase Transitions and Critical Phenomena, edited by C. Domb and J. L. Lebowitz (Academic Press, London, 1987), Vol. 8, p. 1, and references therein.

[2] H. W. Diehl, in Phase Transitions and Critical Phenomena (Ref. [1]), Vol. 10, p. 76, and references therein.

[3] J. L. Cardy, in Phase Transitions and Critical Phenomena (Ref. [1]), Vol. 11, p. 55, and references therein.

[4] D. P. Landau and K. Binder, Phys. Rev. B 41, 4633 (1990).

[5] J. L. Cardy, Nucl. Phys. B: Field Theory Stat. Syst. 240 [FS12], 514 (1984).

[6] J. L. Cardy, Nucl. Phys. B: Field Theory Stat. Syst. 275 [FS17], 200 (1986).

[7] J. L. Cardy, Nucl. Phys. B 324, 581 (1989).

[8] C. Ruge and F. Wagner, Phys. Rev. B 52, 4209 (1995).

[9] M. P. Nightingale and H. W. J. Blöte, Phys. Rev. B 48, 13678 (1993).

[10] K. Binder, D. P. Landau, and M. Muller, J. Stat. Phys. 110, 1411 (2003).

[11] H. W. Diehl, Int. J. Mod. Phys. B 11, 3503 (1997).

[12] M. Campostrini, A. Pelissetto, and E. Vicari, Phys. Rev. E 65, 066127 (2002); Y. Deng and H. W. J. Blöte, ibid. 68, 036125 (2003), and references therein.

[13] L. Onsager, Phys. Rev. 65, 117 (1944).

[14] Y. Deng and H. W. J. Blöte, Phys. Rev. E 67, 066116 (2003).

[15] B. M. McCoy and T. T Wu, Phys. Rev. 162, 436 (1967).

[16] M. E. Fisher and A. E. Ferdinand, Phys. Rev. Lett. 19, 169 (1967)

[17] See, e. g., F. Y. Wu, Rev. Mod. Phys. 54, 235 (1982).

[18] J. L. Black and V. J. Emery, Phys. Rev. B 23, 429 (1981).

[19] R. J. Baxter, J. Phys. A 13, L61 (1980).
[20] B. Nienhuis, in Phase Transitions and Critical Phenomena, (Ref. [13]), Vol. 11, p. 1, and references therein.

[21] B. Nienhuis, J. Phys. A 15, 199 (1982).

[22] A. B. Zamolodchikov, in Fields, Strings, and Quantum Gravity, edited by H. Guo, Z. Qiu, and J. L. H. Tye (Gordon and Breach, New York, 1989).

[23] L. Chim, Int. J. Mod. Phys. A 11, 4491 (1996).

[24] I. Affleck, J. Phys. A 33, 6473 (2000).

[25] A. De Martino and M. Moriconi, Nucl. Phys. B 528, 577 (1998).

[26] G. Feverati, P. A. Pearce, and F. Ravanini, Phys. Lett. B 534, 216 (2002); Nucl. Phys. B 675469 (2003).

[27] R. I. Nepomechie, Int. J. Mod. Phys. A 17, 3809 (2002).

[28] Y. Deng and H. W. J. Blöte, Phys. Rev. E 70, 035107(R) (2004).

[29] B. Nienhuis, A. N. Berker, E. K. Riedel, and M. Schick, Phys. Rev. Lett. 43, 737 (1979).

[30] M. Blume, Phys. Rev. 141, 1517 (1966).

[31] H. W. Capel, Physica (Amsterdam) 32, 966 (1966); 33, 295 (1967).

[32] See, e. g., D. Stauffer and A. Aharony, Introduction to Percolation Theory (Taylor \& Francis, Philadelphia, 1994).

[33] X. Qian, H. W. J. Blöte, and Y. Deng (unpublished).

[34] Y. Deng and H. W. J. Blöte, Phys. Rev. Lett. 88, 190602 (2002).

[35] T. W. Burkhardt and J. L. Cardy, J. Phys. A 20, L233 (1987).

[36] B. Duplantier, Phys. Rev. Lett. 84, 1363 (2000).

[37] Y. Deng, H. W. J. Blöte, and B. Nienhuis, Phys. Rev. E 69, 026123 (2004). 